Supporting Information

\title{
Three Models to Encapsulate Multi-component Dyes into Nanocrystal Pores: A New Strategy for Generating High Quality White Light
}

Xiao-Yuan Liu,,$+\star$ Kai Xing, ${ }^{\dagger}$ Yang Li, ${ }^{\S}$ Chia-Kuang Tsung, ${ }^{*}, \S$ and Jing Li ${ }^{*}, \downarrow, \dagger$

${ }^{\dagger}$ Hoffmann Institute of Advanced Materials, Shenzhen Polytechnic, 7098 Liuxian Blvd, Nanshan District, Shenzhen, 518055, P.R. China

$¥$ Department of Chemistry and Chemical Biology, Rutgers University, 123 Bevier Road, Piscataway, New Jersey 08854, United States

$\S$ Department of Chemistry, Merkert Chemistry Center, Boston College, 2609 Beacon Street, Chestnut Hill, Massachusetts 02467, United States 


\section{Chemicals}

Cetyltrimethylammonium bromide (CTAB, Beantown Chemical, 98\%), 2-methylimidazole (Acros Organics, 99\%), zinc nitrate hexahydrate $\left(\mathrm{Zn}\left(\mathrm{NO}_{3}\right)_{2} \cdot 6 \mathrm{H}_{2} \mathrm{O}\right.$, Alfa Aesar, 99\%), rhodamine B (RB, Sigma-Aldrich, 95\%), fluorescein (F, Acros Organics, pure) and 7-amino-4-(trifluoromethyl)coumarin (C-151, Alfa Aesar, 99\%) were purchased from the mentioned sources and used without further purification. 

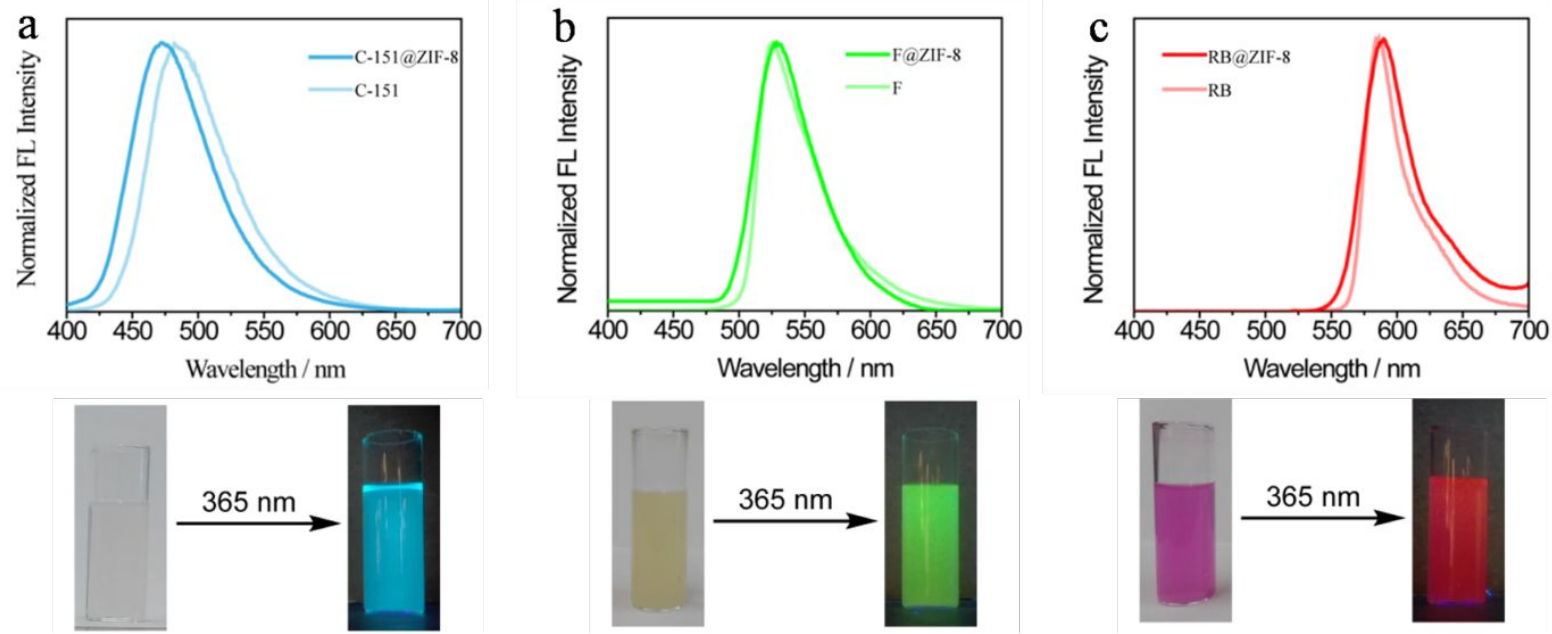

Figure S1. The liquid state fluorescence spectra of (a) C-151@ZIF-8 and C-151, (b) F@ZIF-8 and F and (c) RB@ZIF-8 and RB, and the corresponding photographs of C-151@ZIF-8, F@ZIF-8 and RB@ZIF-8 in methanol solution under daylight (left) and UV light (right). 

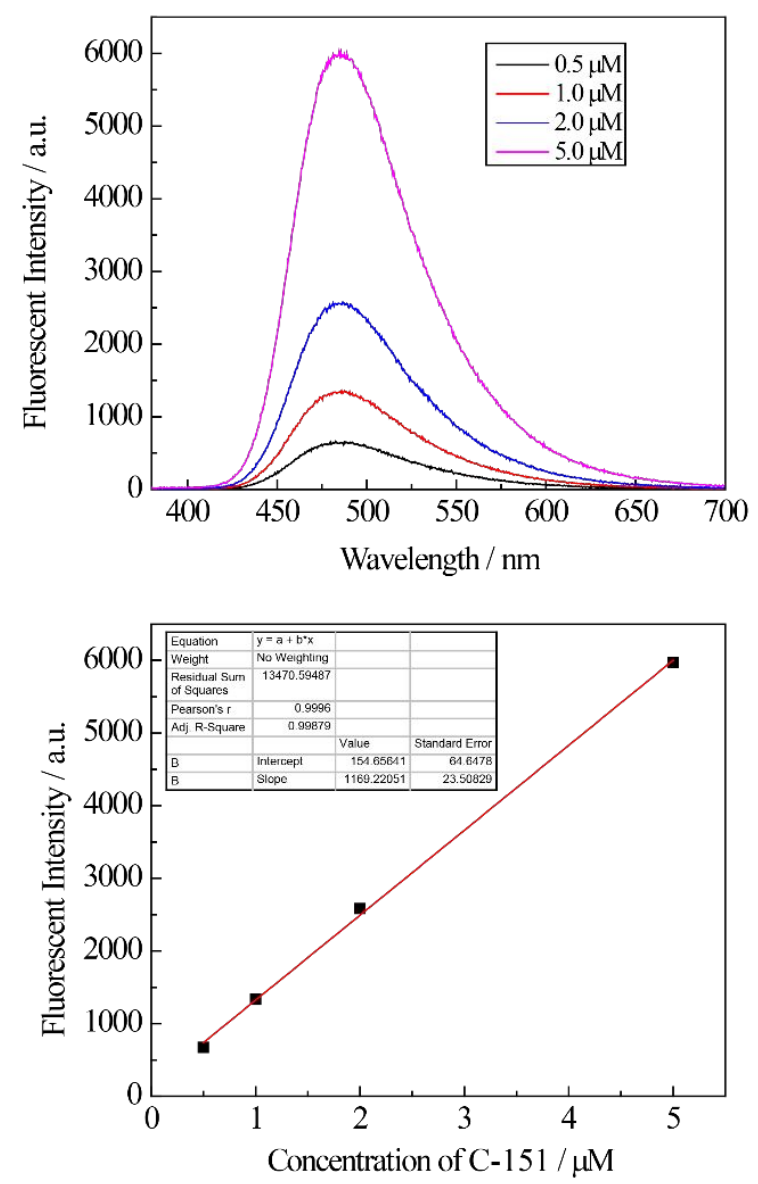

Figure S2. The relationship of concentration and fluorescent intensity of C-151.

The content of C-151 in C-151@ZIF-8 ${ }^{2}$ was calculated as following:

Different amount of C-151@ZIF-8² was dispersed in $2 \mathrm{~mL}$ methanol containing 1 drop of diluted $\mathrm{HCl}$. Then the fluorescent intensity was measured and the content was calculated using the concentration-intensity equation as shown in Figure S2 inset.

Table S1. The related parameters of C-151 in C-151@ZIF-82.

\begin{tabular}{|c|c|c|c|c|}
\hline C-151 solution (mL) & 0.10 & 0.37 & 0.5 & 0.75 \\
\hline Quantity (mg) & 14.0 & 16.1 & 14.0 & 13.0 \\
\hline Fluorescent intensity & 1597 & 5789 & 6267 & 7338 \\
\hline Mass ratio (wt\%) & 0.0040 & 0.0137 & 0.0171 & 0.0217 \\
\hline Quantum yield & 0.267 & 0.419 & 0.208 & 0.166 \\
\hline
\end{tabular}



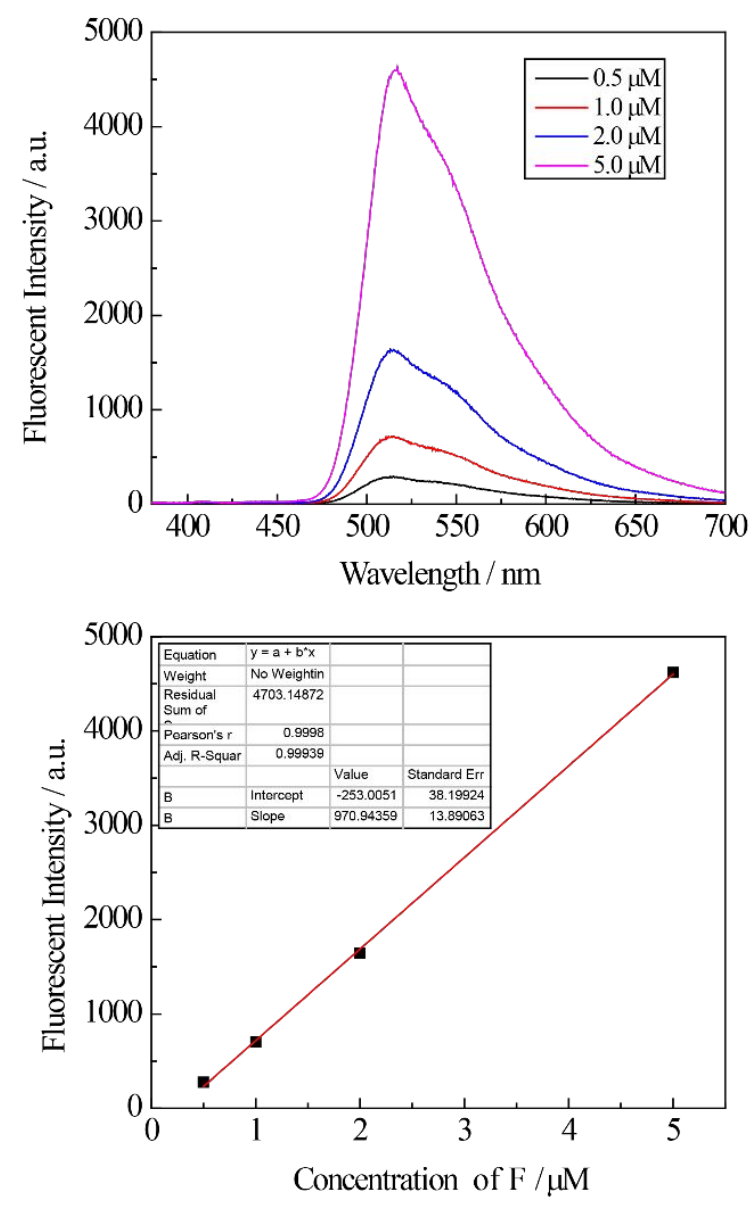

Figure S3. The relationship of concentration and fluorescent intensity of F.

The content of F in F@ZIF-82 was calculated using the same method as C-151.

Table S2. The related parameters of F in F@ZIF- $8^{2}$.

\begin{tabular}{|c|c|c|c|c|}
\hline F solution $(\mathrm{mL})$ & 0.10 & 0.25 & 0.75 & 1.00 \\
\hline Quantity $(\mathrm{mg})$ & 13.3 & 12.9 & 13.4 & 13.8 \\
\hline Fluorescent intensity & 340 & 1101 & 2600 & 3386 \\
\hline Mass ratio (wt\%) & 0.0030 & 0.0072 & 0.0146 & 0.0180 \\
\hline Quantum yield & 0.595 & 0.626 & 0.459 & 0.428 \\
\hline
\end{tabular}



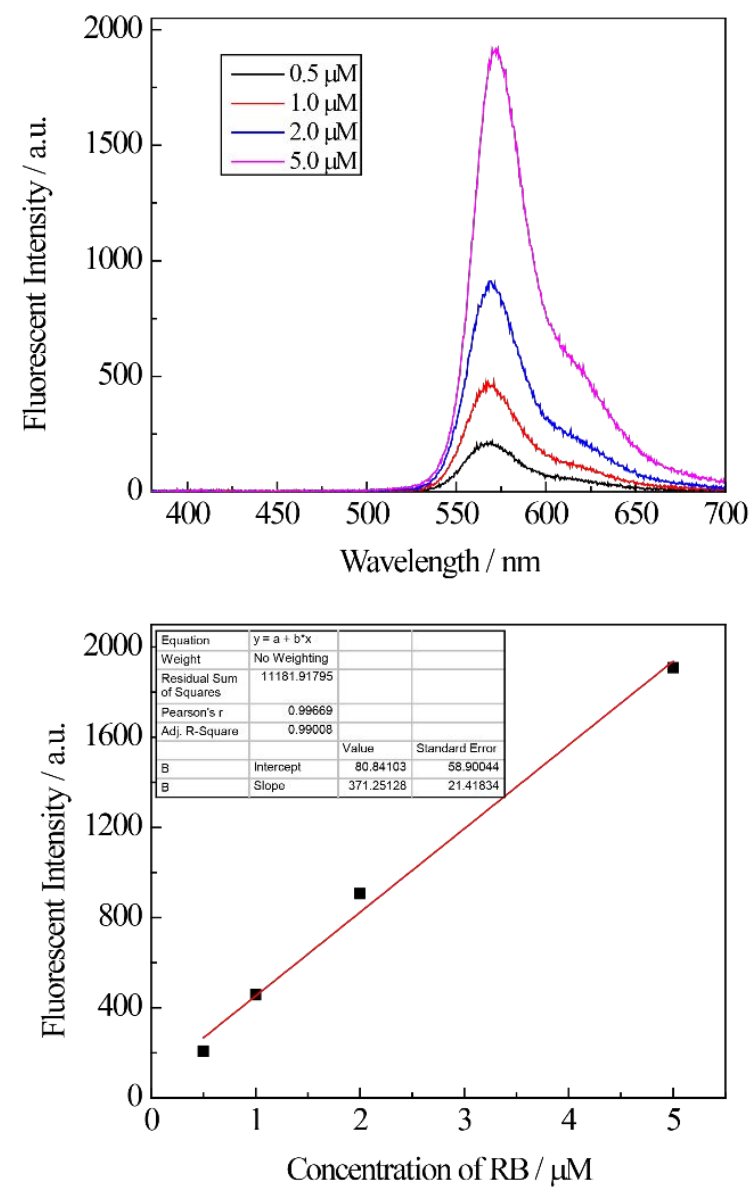

Figure S4. The relationship of concentration and fluorescent intensity of RB.

The content of RB in RB@ZIF- $8^{2}$ was calculated using the same method as C-151.

Table S3. The related parameters of RB in RB@ZIF- $8^{2}$.

\begin{tabular}{|c|c|c|c|c|}
\hline RB solution $(\mathrm{mL})$ & 0.10 & 0.25 & 0.50 & 0.75 \\
\hline Quantity $(\mathrm{mg})$ & 13.5 & 14.0 & 12.1 & 13.4 \\
\hline Fluorescent intensity & 775 & 2104 & 3361 & 4253 \\
\hline Mass ratio (wt\%) & 0.0132 & 0.0372 & 0.0705 & 0.0803 \\
\hline Quantum yield & 0.492 & 0.601 & 0.369 & 0.232 \\
\hline
\end{tabular}



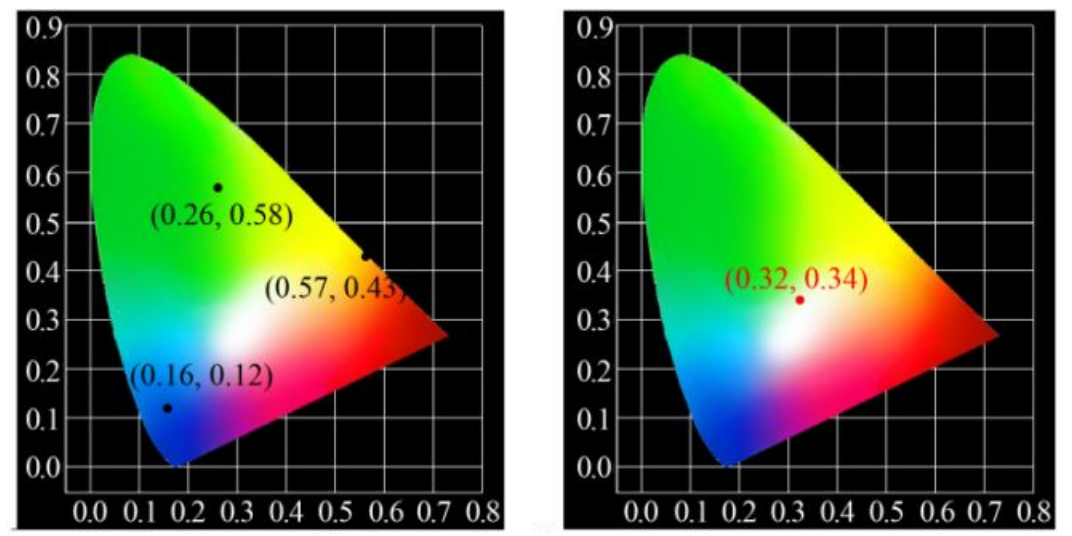

Figure S5. The CIE chromaticity coordinates of C-151@ZIF-8², F@ZIF-8² and RB@ZIF-82 (left) and the corresponding CIE chromaticity coordinates of white light emission (a grinding mixture of 0.0137 wt\% C-151@ZIF-8²,0.0146wt\% F@ZIF-8² and 0.0372wt\% RB@ZIF-82 with mass ratio $2: 1: 3$ ) (right). 

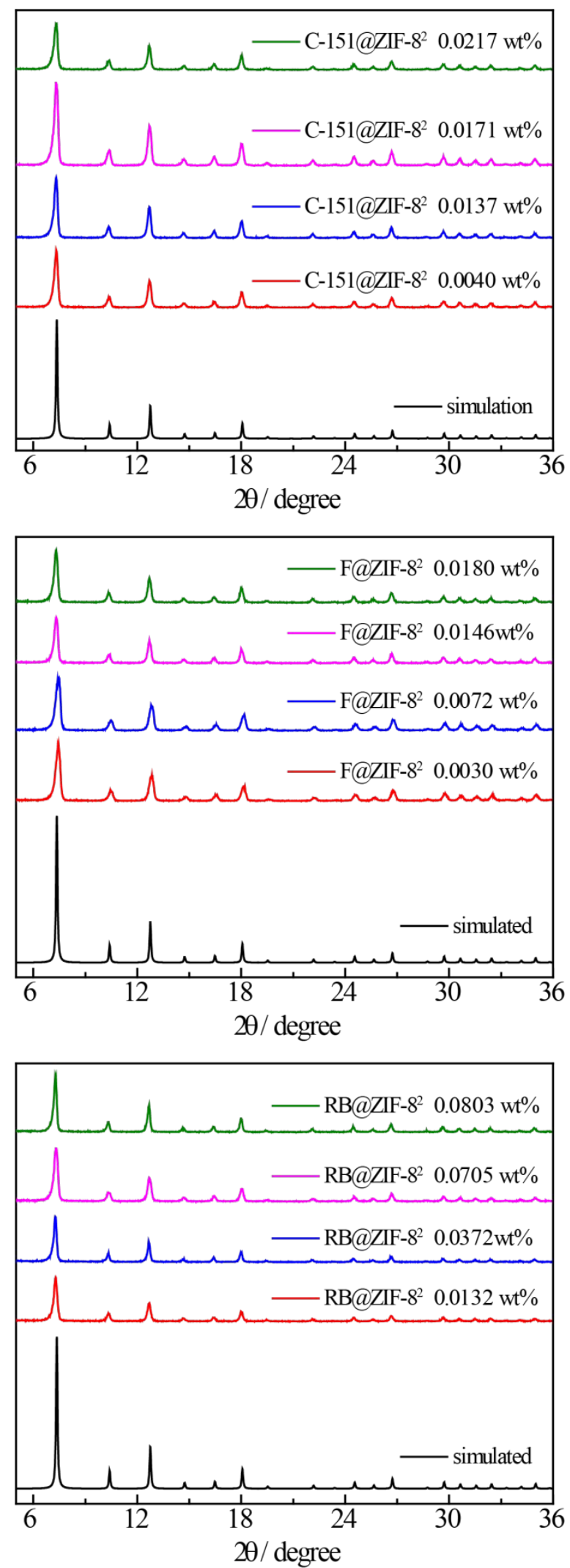

Figure S6. The PXRD patterns of C-151@ZIF-82 (top), F@ZIF-82 (middle) and RB@ZIF-82 (below) with different dye concentrations. 

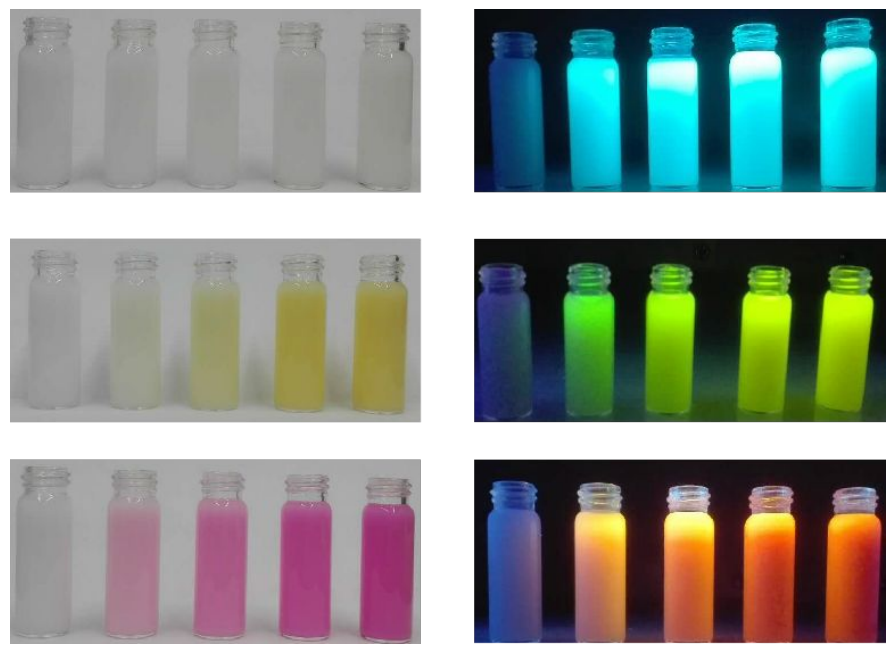

Figure S7. The photographs of methanol solution of C-151@ZIF-82 (top), F@ZIF-82 (middle) and RB@ZIF-82 (below) with different dye concentration under daylight (left) and UV light (right). 

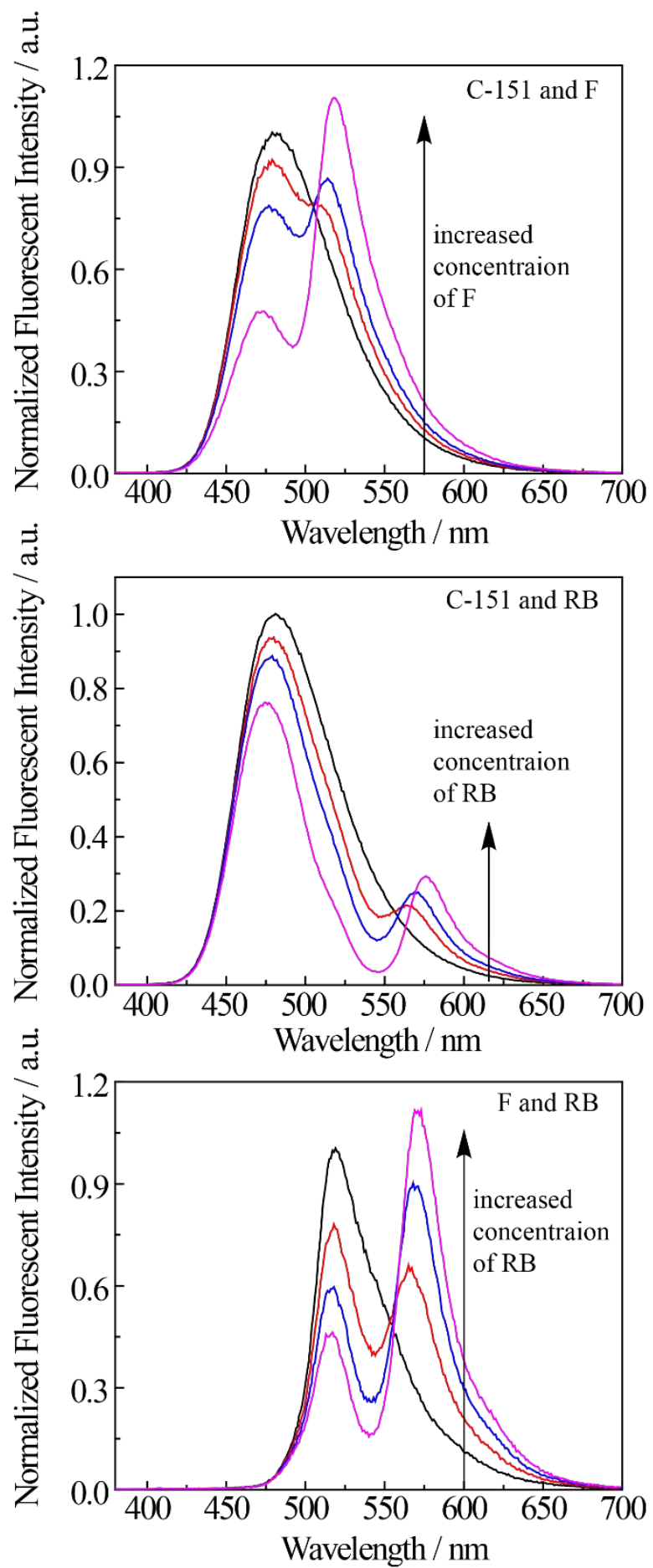

Figure S8. The Fluorescence Resonance Energy Transfer (FRET) measurements between C-151 and F (top), C-151 and RB (middle) and F and RB (below).

As shown in Figure S8, in the case of C-151 and F, the fluorescent intensity of C-151 (donor) exhibits a significant decrease with increase concentration of $\mathrm{F}$ (acceptor). Therefore, it is clear that there exist strong energy transfer between $\mathrm{C}-151$ and $\mathrm{F}$ in methanol solution. The similar results were also observed between C-151 and RB, F and RB. 


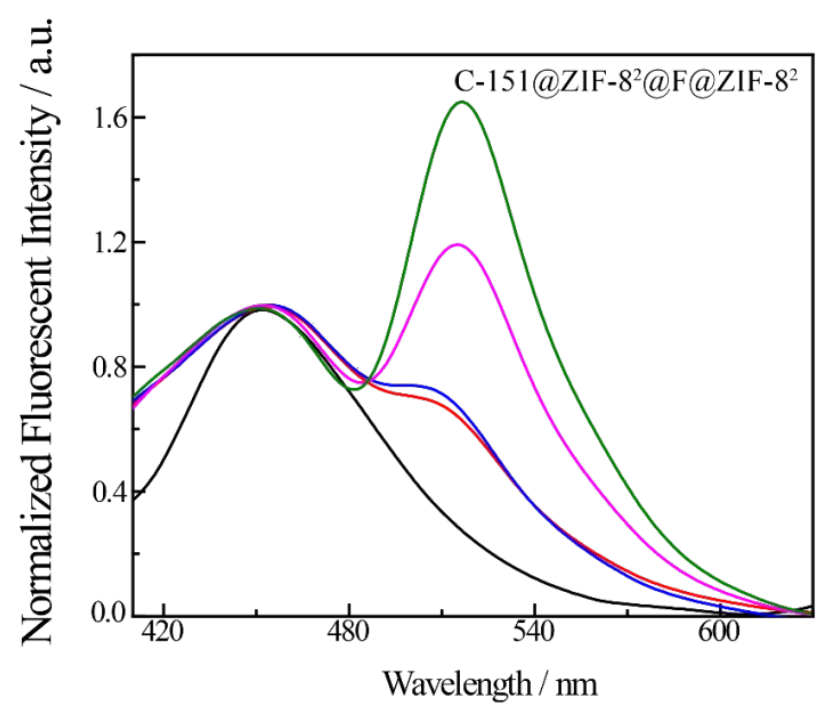

Figure S9. The fluorescence spectra of C-151@ZIF-82@F@ZIF-8 ${ }^{2}$ with increased concentrations of $\mathrm{F}$. 

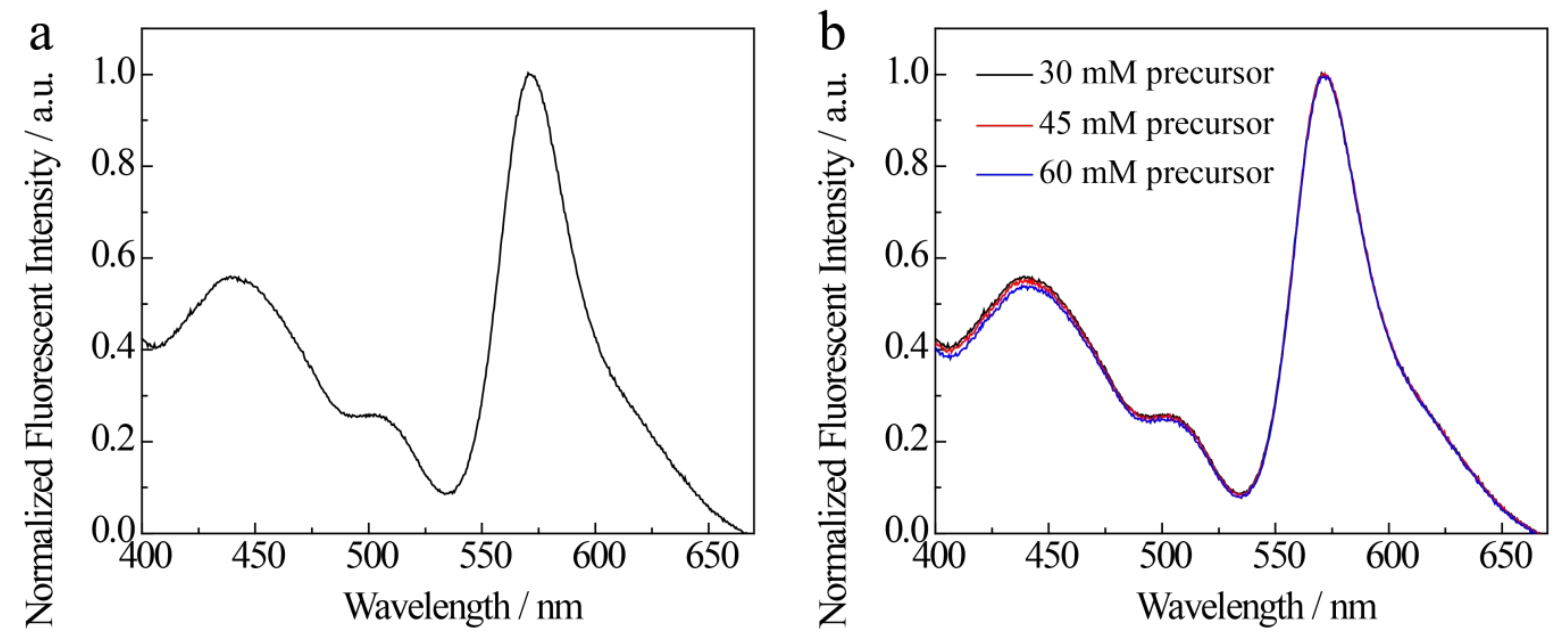

Figure S10. (a) The fluorescence spectrum of RB@ZIF-8@F@ZIF-8²@C-151@ZIF-8² with a CIE chromaticity coordinates of $(0.35,0.33)$. (b) The fluorescence spectra of RB@ZIF-8@F@ZIF-8²@C-151@ZIF-8² with increasing out-shell thickness of ZIF-8. The corresponding precursor concentrations are $30 \mathrm{mM}$ (black), $45 \mathrm{mM}$ (red) and $60 \mathrm{mM}$ (blue), respectively, for the overgrowth of the out-shell ZIF-8. 
a
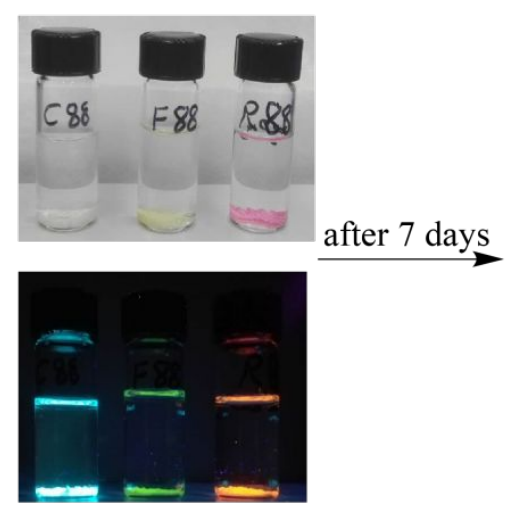

dye@ZIF-8 ${ }^{2}$
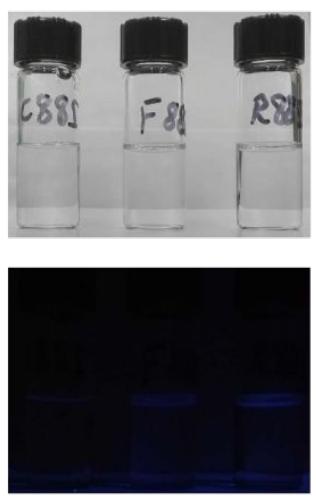

supernatant

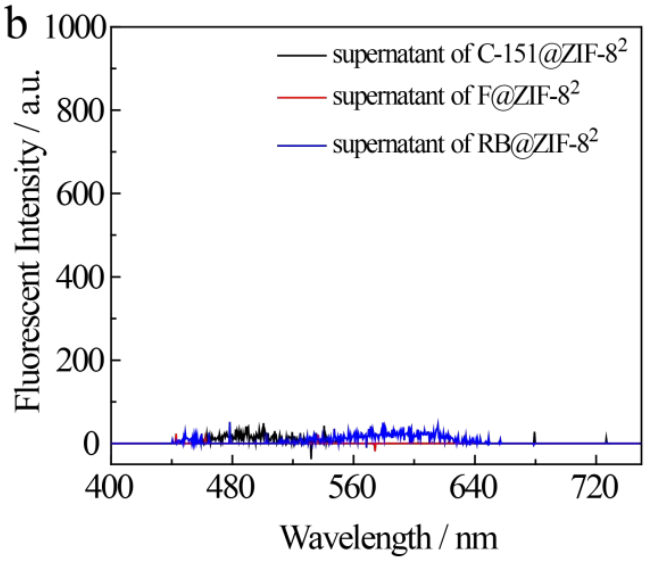

Figure S11. (a, left) The photographs of C-151@ZIF-8², F@ZIF-8² and RB@ZIF-8² soaked methanol solution under daylight (top) and UV light (bottom); (a, right) the photographs of supernatants of C-151@ZIF-82, F@ZIF-8² and RB@ZIF-8² soaked methanol solution after 7 days under daylight (top) and UV light (bottom); (b) the fluorescence spectra of supernatants of C-151@ZIF-8², F@ZIF-8² and RB@ZIF-8² soaked methanol solution after 7 days. 